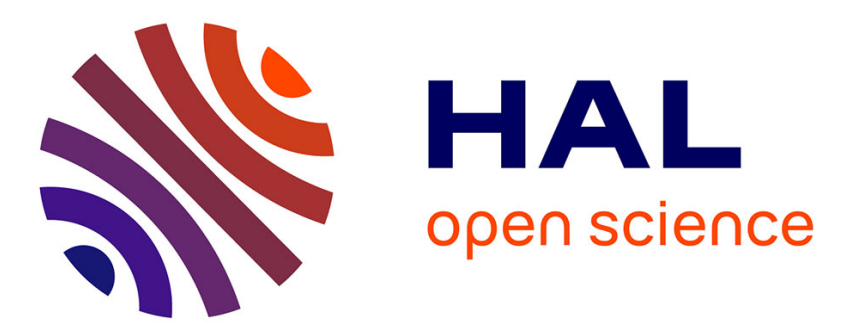

\title{
An Improved STEM/EDX Quantitative Method for Dopant Profiling at the Nanoscale
}

Raghda Makarem, Fuccio Cristiano, Dominique Muller, Pier-Francesco Fazzini

\section{To cite this version:}

Raghda Makarem, Fuccio Cristiano, Dominique Muller, Pier-Francesco Fazzini. An Improved STEM/EDX Quantitative Method for Dopant Profiling at the Nanoscale. Microscopy and Microanalysis, 2020, 26 (1), pp.76 - 85. 10.1017/s1431927619015241 . hal-03097328

\section{HAL Id: hal-03097328 \\ https://hal.laas.fr/hal-03097328}

Submitted on 5 Jan 2021

HAL is a multi-disciplinary open access archive for the deposit and dissemination of scientific research documents, whether they are published or not. The documents may come from teaching and research institutions in France or abroad, or from public or private research centers.
L'archive ouverte pluridisciplinaire HAL, est destinée au dépôt et à la diffusion de documents scientifiques de niveau recherche, publiés ou non, émanant des établissements d'enseignement et de recherche français ou étrangers, des laboratoires publics ou privés. 


\title{
An improved STEM/EDX quantitative method for dopant profiling at the nanoscale
}

November 7, 2019

R. Makarem ${ }^{1}$, F.Cristiano ${ }^{2}$, D.Muller ${ }^{3}$, P.F.Fazzini ${ }^{1}$

1 - LPCNO, Université de Toulouse INSA, CNRS, UPS 135, Avenue de Rangueil, 31077 Toulouse (France)

2 - LAAS-CNRS, Université de Toulouse, CNRS, 7 avenue du colonel Roche, F-31400 Toulouse, France

3- ICube Laboratory, Université de Strasbourg and CNRS, B.P. 20, 67037 Strasbourg Cedex, France

\begin{abstract}
In this paper, an improved quantification technique for STEM/EDX measurements of $1 \mathrm{D}$ dopant profiles based on the Cliff-Lorimer equation is presented. The technique uses an iterative absorption correction procedure based on density models correlating the local mass density and composition of the specimen. Moreover, a calibration and error estimation procedure based on linear regression and error propagation is proposed in order to estimate the total measurement error in the
\end{abstract}


dopant density. The proposed approach is applied to the measurement of As profile in a nanodevice test structure. For the calibration, two crystalline Si specimens implanted with different As doses have been used and the calibration of the Cliff-Lorimer coefficients has been carried out using Rutherford Back Scattering measurements. The As profile measurement has been carried out on a FinFET test structure, showing that quantitative results can be obtained in the nanometer scale and for dopant atomic densities lower than 1\%. Using the proposed approach, the measurement error and detection limit for our experimental setup are calculated and the possibility to improve this limit by increasing the observation time is discussed.

\section{Introduction}

One of the key issues for the miniaturization of semiconductor nanodevices is the precise control of their doping (Vandervorst et al., 2014). In new generation devices, the doping spatial distribution must be controlled with a precision higher than $1 \mathrm{~nm}$ while atomic concentrations below $1 \%$ have to be measured. This is a particularly critical issue for the characterization of nanodevices and 3D transistors (Colinge, 2008), where standard techniques such as as Secondary Ion Mass Spectroscopy (SIMS) cannot be used. This calls for the use of high resolution techniques. Scanning transmission electron microscopy (STEM) associated with Energy Dispersive X-ray spectroscopy (EDX) is an excellent candidate due to its versatility (several dopants can be mapped) and its high spatial resolution. Moreover, the current availability of probe spherical aberration correction, high brightness sources, and high angle 
silicon drift detectors (SDD) make this technique even more attractive since a high signal to noise ratio can be obtained with the characteristic resolution of STEM imaging techniques. Atomic elemental mapping of alloys can be obtained using commercially available microscopes (Kothleitner et al., 2014).

In spite of all these advances, using STEM/EDX to obtain quantitative information on the doping distribution in a nano-device is still a challenging task. Quantitative analysis is complicated by the presence of measurement artifacts (Goldstein et al., 2018)(Williams \& Carter, 2009) that can safely be ignored for high concentrations impurities, but become critical for low concentration dopants (concentration lower than 1\%) since the artifact contribution can be of the same order of magnitude as the signal to be measured. More specifically the artifacts complicating doping profiling with STEM/EDX can be divided into two categories:

- The artifacts arising from the detector design such as escape peaks, sum peaks, peak deformations due to partial charge collection and the absorption of X-rays in the detector surface and window (Goldstein et al., 2018). The effect of these artifacts are negligible in modern SDD devices, thanks to the careful design minimizing the size of the dead layers in the active surface and the fact that the constructors take into account the absorption in the detector using a quantum efficiency function (Alvisi et al., 2006).

- The artifacts arising from the physical interactions (absorption, fluorescence ...) of X-rays emitted in the specimen with the specimen itself and other elements in the column (Williams \& Carter, 2009). This 
problem is unavoidable and can affect the accuracy of the obtained results, especially when measuring dopant concentration. Particular attention must be paid to the effect of the absorption of X-rays in the specimen. A rigorous treatment of the effects of self-absorption on the measured composition calls for the employ of an iterative approach since the absorption effects clearly depend on the specimen composition (Watanabe et al., 1996).

In this paper we will present a modified Cliff-Lorimer (Cliff \& Lorimer, 1975) method in order to deal with the second kind of artifacts and to obtain quantitative dopant profiling in a nanoscale device structure. The method has been applied on specimens prepared by Focused Ion Beam, in order to reduce the influence of secondary X-rays produced by fluorescence or back scattered electrons, and is based on the iterative correction of X-rays absorption effects in the specimen. In order to obtain reliable results the k-factors have been calibrated using Rutherford Back Scattering (RBS) measurements and the total experimental error has been calculated using standard error propagation techniques. The method has then been applied to the measurement of As dopant profiles in a FinFET structure,

The main novelties of this approach is the use of RBS data to calibrate the $\mathrm{k}$ - factors and that the specimen local density has been calculated using physical models. The knowledge of the local density is used to correct the absorption effects without any in-situ measurement of the electron beam current, which is an advantage with respect to other techniques such as the $\zeta$-factor (Watanabe \& Williams, 2006) or cross-section method (MacArthur et al., 2016). The main difference between this techniques and other tech- 
niques not requiring an in-situ measurement is that specific peaks family can be selected for each element (while at least two must be used in the technique proposed by Morris et al. (1979) and Qiu et al. (2013)) and there is no need to use several specimen tilt angles (as in the technique proposed by Morris et al. (1979)). The main disadvantage of this approach when compared with the other techniques is that the specimen must have a constant thickness, that the thickness must be measured with other techniques, and that no direct information can be obtained on the specimen density.

\section{Materials and Methods}

In this section the experimental techniques and the data analysis methods used in this work will be presented.

First of all, the specimens that have been used for the calibration of the $\mathrm{k}$-factors and for doping measurements will be described, then some experimental details on the STEM/EDX measurements will be given and, finally, the techniques we used for data quantification will be detailed.

\subsection{Description of the samples}

To be able to measure arsenic doping concentration in silicon nanodevices, we have designed some calibration specimens obtained by ionic implantation of arsenic in monocrystalline silicon wafers. Two specimens were used to check the precision of the calibration on different concentrations. Both were implanted using an acceleration voltage of $21 \mathrm{KeV}$ but with a different dose amounting, respectively, to $9.4 \cdot 10^{15} \mathrm{~cm}^{-2}$ and $1.8 \cdot 10^{15} \mathrm{~cm}^{-2}$. It has to be 
noted that due to the high dose, the implanted wafers are amorphized in the implantation region. The implantation parameters have been chosen by simulating the implantation profile using the SRIM (Stopping and Range of Ions in Matter) Monte Carlo simulation software (Ziegler \& Biersack, 1985). The simulation results corresponding to the two different doses are shown in Figure 1(a). It can be noted that the value of the concentration peak amounts to $5 \cdot 10^{21} \mathrm{~cm}^{-3}$ (equivalent to $10 \%$ atomic As concentration) for the higher dose implant and to $10^{21} \mathrm{~cm}^{-3}$ (2\% atomic As concentration) for the lower dose implant.

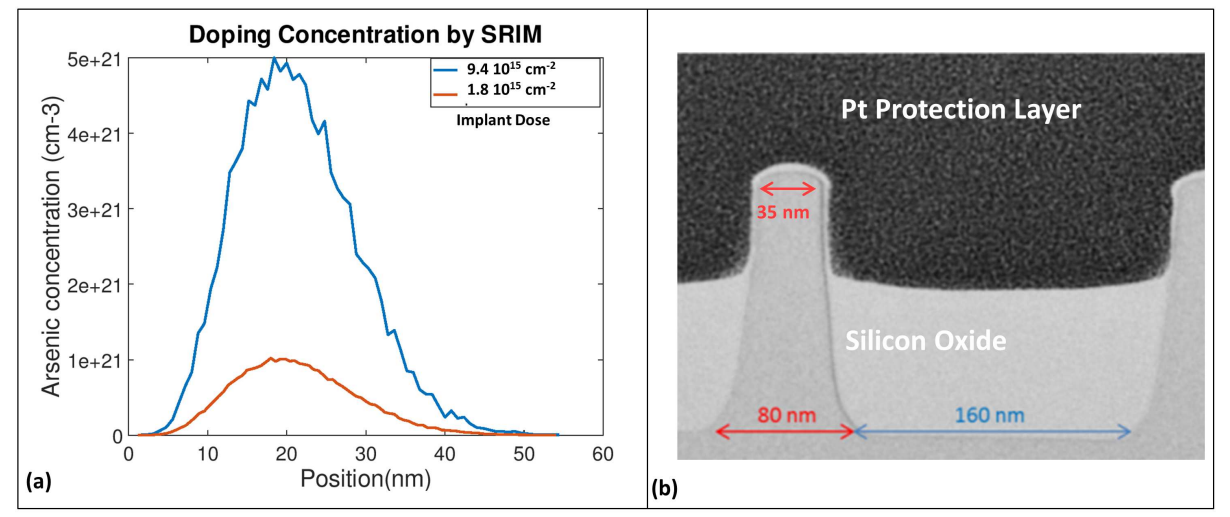

Figure 1: (a) Implanted doping distribution simulated with SRIM for As implant in silicon with an energy of $21 \mathrm{keV}$; (b)Bright field STEM image of the cross section of the FinFET sample.

An absolute measurement of the arsenic concentration of the implanted samples has been obtained in bulk specimens by Rutherford backscattering spectroscopy (RBS) measurements (Chu, 1978) using $\mathrm{He}^{+}$ions accelerated at $1.5 \mathrm{MeV}$. The detector resolution and solid angle have been obtained on a $\mathrm{SiO}_{2}$ reference specimen using a detector collection angle of $10^{\circ}$. For the As concentration measurements an elevation angle of $60^{\circ}$ has been used. 
By using RBS, the atomic concentration profiles (atoms $/ \mathrm{cm}^{3}$ ) of arsenic in the two calibration specimens have been obtained. The final error on the concentration measurement is in the order of $10 \%$ while the spatial resolution of the technique is $4 \mathrm{~nm}$. The RBS results were used to calibrate the $\mathrm{k}$-factors, as explained in section 2.3.3. Since the resolution of the RBS measurements is comparable with the resolution obtained by STEM/EDX, the alignment of the two set of data for the calibration can be easily obtained by aligning the As concentration peaks.

To show the applicability of the proposed measurement technique to nanodevices, a FinFET test structure, with a channel width of $35 \mathrm{~nm}$ and a height of $100 \mathrm{~nm}$ has been used (see Figure 1(b)). In this specimen an As plasma implant followed by a high temperature thermal annealing (higher than $900^{\circ} \mathrm{C}$ ) have been used to obtain a conformal distribution (Vandervorst et al., 2014) of the doping profile in the source and drain regions of the device.

\subsection{Specimen preparation and TEM observations}

TEM observations have been performed on cross sectional lamellas fabricated using a FEI Helios 600i dual beam focused ion beam (FIB) (Ke et al., 2009). A lift-out procedure has been used to transfer the specimen on a supporting copper grid. A platinum layer has been deposited onto the wafer surface before the creation of the lamella to minimize curtaining effects. To avoid any kind of ionic damage near the surface, platinum deposition has been carried out in two consecutive steps by using electron beam-induced deposition followed by ion beam-induced deposition. There are several advantages in 
using FIB specimen preparations for EDX analysis. As discussed in the introduction, the use of a lamella reduces the emission of secondary X-rays by fluorescence and the backscattering in comparison with specimens prepared by other techniques like mechanical grinding followed by ion thinning (see figure 2). Moreover, the use of FIB guarantees that the specimen thickness is constant all over the observation region, located near to the capping $\mathrm{Pt}$ layers. Finally, FIB preparation has been used in the case of the FinFET structure to select a precise specimen region such as the implanted regions shown in figure 1. Electron energy loss spectroscopy (Malis et al., 1988) has been used to estimate the value and uniformity of the specimen thickness. For the three specimens a value of $150 \mathrm{~nm}$ with negligible variations in the observation region has been obtained.

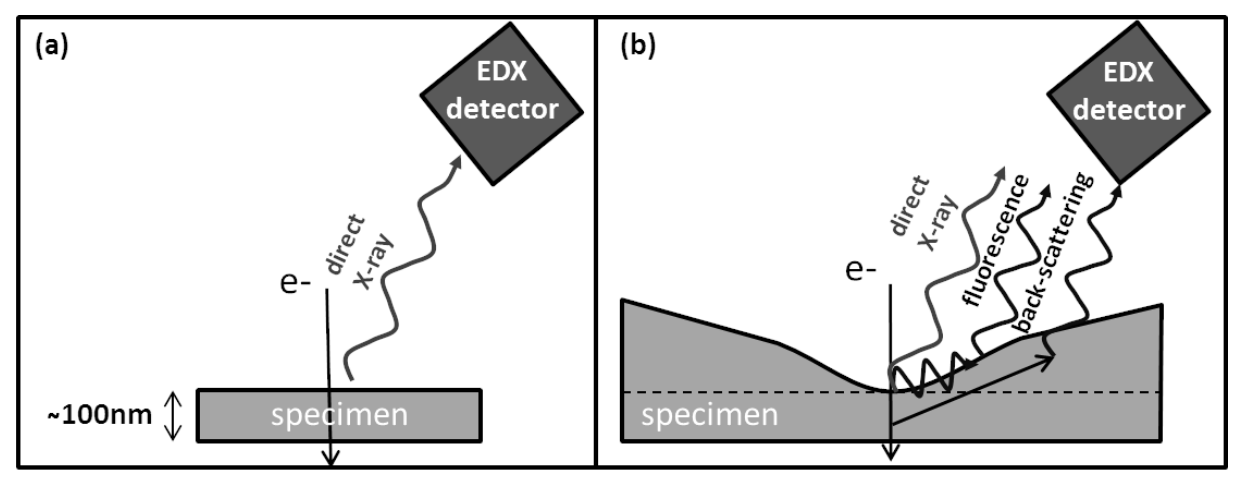

Figure 2: Schematic illustration of the influence of the specimen geometry on EDX artifacts (secondary X-rays created by fluorescence and backscattered electrons) in specimens prepared using: a) FIB. b) mechanical thinning.

The STEM/EDX measurements have been carried out using a JEOL ARM cold FEG microscope equipped with a probe spherical aberration corrector. For the EDX measurements, a CENTURIO-X detector with a take-off angle of 24.3 degrees and a collection angle of 0.98 steradians has been used. 
In order to exploit the 1D geometry of the observed samples the specimens have been oriented as in Figure 3. The reason of this choice is that, if we consider the detector size as negligible, the X-rays reach the detector passing through a slice of homogeneous density.

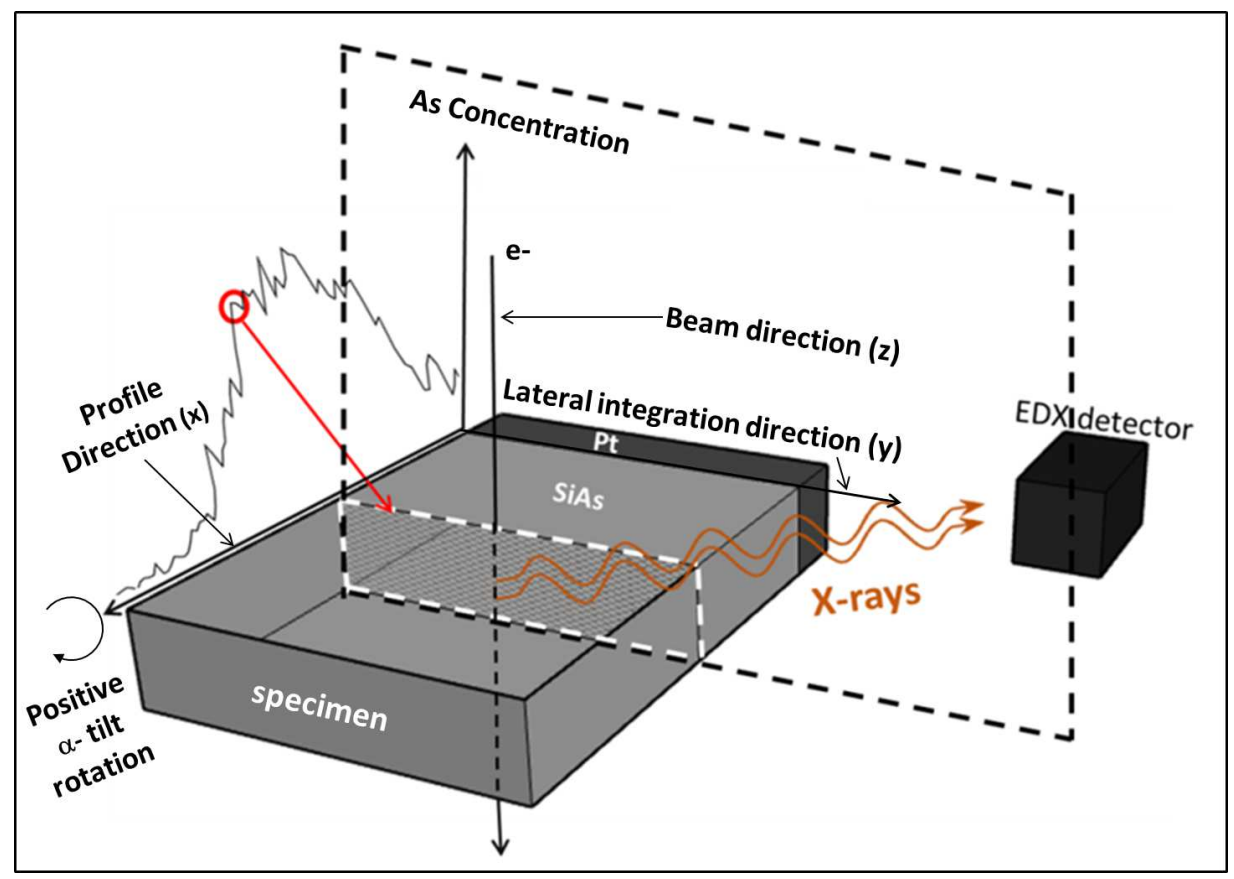

Figure 3: Schematic illustration of the detector position and of X-ray path, when the detector is placed on a plane perpendicular to the variation direction of the doping profile.

2D STEM/EDX maps of the observed region have been used to measure the dopant profile in the $x$ direction. First of all an EDX spectrum has been acquired for each pixel of the 2D map. Then the JEOL Analysis Station program $^{1}$ has been used to subtract the Bremsstrahlung background noise pixel by pixel and to calculate the integrated intensities of the characteristic peaks for the various elements in order to obtain $2 \mathrm{D}$ intensity maps. The uni-

\footnotetext{
${ }^{1}$ See the website https://www.jeol.co.jp/en/products/detail/JED-2300_2300F.html for more information
} 
dimensional intensity profiles have then been obtained from the $2 \mathrm{D}$ intensity maps by lateral integration in the $y$ direction (see figure 3). Observations have been carried out for all observed specimens in the same experimental conditions. The FIB lamella was placed on the side of the central finger of a three post Omniprobe lift-out grid $^{2}$. A JEOL analytical double tilt holder with material removed on the detector side, designed to minimize penumbra effects, was used. The z position of the holder was adjusted in order to have the specimen at eucentric eight and a tilt angle of $10^{\circ}$ around the holder axis ( $\alpha$-tilt, see figure 3 ) has been used in order to further minimize penumbra effects. In the particular case of $1 \mathrm{D}$ objects, this does not induce any deformation of the concentration profile.

\subsection{Quantification Method}

As already mentioned, for the quantification of the specimen composition we have used an approach based on Cliff-Lorimer factors. Data treatment has been carried out using the scientific programming language GNU Octave ${ }^{3}$.

The starting point for our quantification method is the Cliff and Lorimer equation giving the mass fractions of a specific element $i$ (Williams \& Carter, 2009):

$$
f_{i}^{M}=\frac{k_{i: F_{i}, F_{i_{0}}} I_{i: F_{i}}}{\sum_{j=1}^{N_{A}} k_{j: F_{j}, F_{i_{0}}} I_{j: F_{j}}}
$$

where $N_{A}$ is the total number of elements in the sample $f_{i}^{M}$ is the mass fraction of the $\mathrm{i}^{\text {th }}$ element and $I_{i: F_{i}}$ is the intensity of the characteristic peak associated to the $F_{i}$ family and $k_{i: F_{i}, F_{i}}$ is the k-factors relative to the element

\footnotetext{
${ }^{2}$ See for example http://www.agarscientific.com/fib/grids-for-fib/3-post-lift-out-grids

${ }^{3}$ See the website https://www.gnu.org/software/octave/ for more information
} 
$i$ and a common reference element $i_{0}$ that, by definition, has a unitary k-factor $\left(k_{i_{0}: F_{i_{0}}, F_{i_{0}}}=1\right)$.

The total intensity $I_{i: F_{i}}$ of the family $F_{i}$ for a specific element is obtained by adding the intensities $I_{i: F_{i}, k}$ of the $N_{F_{i}}$ lines $(K \alpha, K \beta \ldots)$ belonging to the family chosen for the element $i$. Each specific line of the family is associated to an energy $E_{i: F_{i}, k}$. While mass fractions are easily obtained by EDX, atomic concentrations (in atoms $/ \mathrm{cm}^{3}$ ) are customary in semiconductor literature. The following formula can be used to convert mass fractions to atomic fractions $f_{i}^{A}$ or concentrations $c_{i}^{A}$ :

$$
f_{i}^{A}=\frac{f_{i}^{M} / m_{i}}{\sum_{j} f_{j}^{M} / m_{j}}, c_{i}^{A}=\frac{\rho^{M} f_{i}^{M}}{m_{i}} N_{A V}
$$

where $\rho^{M}$ is the mass density of the specimen, $m_{i}$ is the atomic mass of the $i$ element and $N_{A V}=6,02214076 \cdot 10^{23} \mathrm{~mol}^{-1}$ is the Avogadro number.

\subsubsection{Iterative absorption calculation}

In a homogeneous material of constant thickness, the peak intensity $I^{*}(E)$ after self-absorption of X-rays of energy $E$ and initial intensity $I(E)$ can be calculated as follows (Williams \& Carter, 2009) :

$$
I^{*}(E)=\frac{1-e^{-\mu(E) \rho^{M} t / \sin (\alpha)}}{\mu(E) \rho^{M} t / \sin (\alpha)} I(E)=T \cdot I(E)
$$

where $\mu(E)$ is the mass absorption coefficient of the material, $t$ is the material thickness, $\rho^{M}$ is its mass denisty, $\alpha$ is the take-off angle which is equal to $34.3^{\circ}$ in our case. In the previous formula we have also defined the 
transmission factor $T$ to simplify further notation. For an alloy, $\mu(E)(($ Eibl, 1993)) must be calculated as follows:

$$
\mu\left(E,\left\{f_{i}^{M}\right\}\right)=\sum_{i=1}^{N_{A T}} \mu_{i}(E) f_{i}^{M}
$$

where $\mu_{i}(E)$ is the mass absorption coefficient associated to the $\mathrm{i}^{\text {th }}$ atom. In this work the value of $\mu_{i}(E)$ for the various elements has been calculated using the EPQ library implementation of the model proposed in (Heinrich, 1986). For that, the Java interface features of Octave have been used to access the EPQ library routines.

As already observed, equation 3 can only be used for homogeneous specimens of constant thickness. In our case both conditions hold because of the choice of the specimen orientation (see figure 3) and of the FIB specimen preparation (section 2.1) used in this work. Equation 3 can be used to implement an iterative approach provided that the relation between the local density and the local composition (expressed in the form $\rho^{M}\left(\left\{f_{i}^{M}\right\}\right)$ ) is known. This is the approach used in this paper and various models that can be used to evaluate $\rho^{M}$ are discussed in section 2.3.2). In this case the transmission factor is a function of the energy and the mass fractions and can be written as $T\left(E,\left\{f_{i}^{M}\right\}\right)$.

For the iterative procedure the initial mass fractions $\left\{f_{i}^{M}\right\}_{0}$ are calculated from the measured intensities by neglecting absorption and are used as a starting point. For each iteration the transmission factor is calculated using the set of mass fractions $\left\{f_{i}^{M}\right\}_{n}$ obtained in the previous iteration. For a peak located at an enrgy $E$ this can be written: 


$$
[T(E)]_{n}=T\left(E,\left\{\left[f_{i}^{M}\right]_{n-1}\right\}\right.
$$

At each iteration the value of the absorption corrected intensities is calculated as follows $[I(E)]_{n}=I_{E}^{*} /[T(E)]_{n}$ and the corresponding absorption corrected mass fractions are evaluated from the corrected intensities using equation 1.

This same technique is applied at each position $x$ of the profile and for each intensity line of the selected peak families. At the end of the iterations the absorption corrected mass fractions $\widetilde{f}_{i}^{M}(x)$ are obtained. The stopping criterion is that the relative variation of the mass fractions between two subsequent iterations is below a tolerance $\epsilon$ that can be chosen by the user $\left(\left|\left(f_{i}^{M}\right)_{n}-\left(f_{i}^{M}\right)_{n-1}\right| /\left(f_{i}^{M}\right)_{n}<\epsilon\right)$.

\subsubsection{Mass density calculation}

For a given material, the application of the iterative procedure requires to establish the appropriate relation between the specimen local mass density $\rho^{M}(x)$, and the local elements mass fractions $f_{i}^{M}(x)$. In this section two formulas will be derived for the amorphous SiAs alloy (a-SiAs) used for the calibration and for the As implanted crystalline silicon (c-SiAs) composing the FinFET test structure.

To derive the formula for a-SiAs, it must be noted that the covalent radii for silicon and arsenic have a close value, $R_{S i}=(111 \pm 2) p m$ and $R_{A s}=(119 \pm 4) p m$, while the value of their atomic masses greatly differs $\left(m_{S i}=28.0855 \mathrm{~g} \cdot \mathrm{mol}^{-1}\right.$ and $\left.m_{A s}=74.9216 \mathrm{~g} \cdot \mathrm{mol}^{-1}\right)$. Hence, a possible 
approximation to evaluate the density consists in neglecting the difference in atomic radius, so that the mass density can be easily calculated starting from the mass density of amorphous $\mathrm{Si}$ and evaluating the mass difference obtained when an As atom replaces a $\mathrm{Si}$ atom. If we define the local atomic fractions for the two elements $f_{A s}^{A}(x)$ and $f_{S i}^{A}(x)$ and we take into account that for a binary alloy we have $f_{S i}^{A}(x)=1-f_{A s}^{A}(x)$ we can write:

$$
\rho_{S i A s}^{M}(x)=\rho_{a-S i}^{M}\left[1+\left(\frac{m_{A s}}{m_{S i}}-1\right) f_{A s}^{A}(x)\right]
$$

Where $\rho_{a-S i}^{M}=2.285 \mathrm{~g} \cdot \mathrm{cm}^{-3}$ is the amorphous Si mass density(Custer et al., 1994). This equation is a function of the As atomic fraction but it can be easily expressed as a function of the mass fractions using equation 2 :

$$
\rho_{a-S i A s}^{M}=\rho_{a-S i}^{M}\left[1+\frac{\left(m_{A s}-m_{S i}\right) f_{A s}^{M}(x)}{m_{A s}-\left(m_{A s}-m_{S i}\right) f_{A s}^{M}(x)}\right]
$$

We will refer to this approximation in the following as the "same radius approximation". The problem of this approach is that it overestimates the mass density of a-SiAs for high arsenic concentrations as shown in figure 4 .

A better approximation can be obtained by using a formula that has been originally derived for solids that do not present any kind of ordering (also known as ideal amorphous solids (IAS)) Stachurski (2015):

$$
\rho_{a-S i A s}^{M}=\rho_{a-A s}^{M} f_{A s}^{V}+\rho_{a-S i}^{M} f_{S i}^{V}
$$

where $\rho_{S i}^{M}$ and $\rho_{A s}^{M}$ are the mass densities of amorphous Si and amorphous As respectively while $f_{A s}^{V}$ and $f_{S i}^{V}$ are the volume fractions of the two elements. 


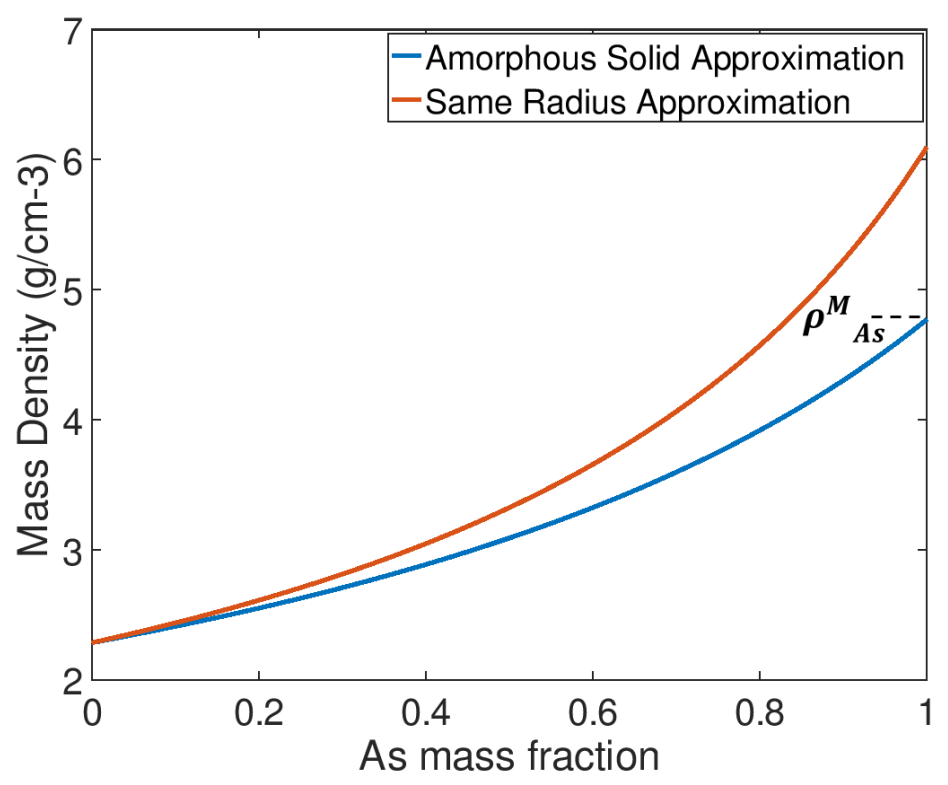

Figure 4: Mass density a function of the As mass fraction calculated with two different approximations. The experimentally determined value of the mass density $\rho_{A s}^{M}$ of amorphous arsenic is shown in the plot.

We will refer to this approximation in the following as the "amorphous solid approximation". The main hypothesis behind the derivation of this formula is that the volume occupied by an atom of each element does not depend on the alloy concentration. A direct consequence of this hypothesis is that the structure around an individual atom must not depend on the alloy concentration.

The atomic arrangement in amorphous As and amorphous Si is very similar. This can be shown by evaluating the ratio $s=V^{S} / V^{A}$ between the atomic volume $V^{A}$ and the atom size $V^{S}$. The volume $V^{A}$ is by definition the inverse of the atomic density $\rho^{A}=\rho^{M} / m$ of the material while the atom size can be calculated by using a spherical approximation $\left(V^{S}=4 / 3 \pi r^{3}\right)$ 
and the covalent radius $r$. The values of $s$ for amorphous silicon and amorphous arsenic can be calculated by using the value of a-As and a-Si densities reported in literature $\left(\rho_{a-A s}^{M}=4.7 \mathrm{~g} / \mathrm{cm}^{3}\right.$, see (Greaves et al., 1979)). The obtained values $\left(s_{S i}=0.28, s_{A s}=0.26\right)$ are close, confirming that the packing of the two structures is almost the same. It can then be assumed that the volume occupied by $\mathrm{Si}$ and As atoms is almost the same in SiAs for any concentration value which is precisely the assumption used to derive equation 8. Using the same reasoning it can be shown that the "amorphous solid approximation" also holds for pseudomorphic alloys such as SiGe. For this kind of structures the crystalline structure of pure and binary alloys are exactly the same (for example crystalline $\mathrm{Si}, \mathrm{Ge}$ and $\mathrm{SiGe}$ all have a diamond structure). Moreover the variation of the lattice parameter with concentration is on the order of some percent. It can then again be assumed that the atomic volume does not vary with the alloy concentration and equation 8 can be used. This example is particularly important since in the case of SiGe the validity of equation 8 can be proved by a direct calculation of the alloy density based on the alloy crystal structure and Vegard's law, which is an excellent approximation of the lattice parameter for SiGe alloys (Kasper et al., 1995).

The volume fractions used in equation 8 can be expressed as a function of the mass fractions as follows:

$$
f_{i}^{V}=\frac{f_{i}^{M}}{\rho_{i}^{M}} / \sum_{j} \frac{f_{j}^{M}}{\rho_{j}^{M}}
$$


Then we finally obtain the equation relating the alloy mass density to the mass fractions measured by EDX:

$$
\rho_{a-S i A s}^{M}=\left(\frac{f_{A s}^{M}}{\rho_{a-A s}^{M}}+\frac{f_{S i}^{M}}{\rho_{a-S i}^{M}}\right)^{-1}
$$

The density function obtained using the "amorphous solid approximation" is shown in figure 4 . It can be observed that this approximation gives better results for high arsenic concentrations while the two approximations are almost equivalent for low As concentrations (see figure 4). Equation 10 has been used to quantify As doping in a-SiAs.

Finally it is important to note that the "same radius approximation" can be used for low doped crystalline Si since in the case the alloy consists in a almost unperturbed Si lattice where some Si atoms are replaced by As atoms. In this case the expression of the density is given by equation 7 where $\rho_{a-S i}^{M}$ is replaced by the density of crystalline Si $\rho_{c-S i}^{M}=2.32 \mathrm{~g} / \mathrm{cm}^{3}$ (Stachurski, 2015). This density equation has been used to quantify As doping in the FinFET test structure.

\subsubsection{Model calibration and error estimation}

In this section we will discuss the calibration procedure used for the k-factors and how we have calculated the total error on the composition measurement. First, for the comparison between EDX rand RBS results, it is necessary to transform the experimental arsenic atomic density concentrations $c_{A s / R B S}^{A}(x)$ 
measured by RBS into mass fractions. This is the best strategy to use since in this way the k-factors can be obtained by weighted linear regression.

Equations 2 and 10 can be combined to obtain the value of the arsenic mass fractions measured by RBS:

$$
f_{A s / R B S}^{M}=\frac{m_{A s} c_{A s / R B S}^{A}(x)}{N_{A V} \rho_{S i}^{M}+m_{A s} c_{A s / R B S}^{A}(x)\left(1-\rho_{S i}^{M} / \rho_{A s}^{M}\right)}
$$

The value of the k-factors for a specific peak family $F_{A s}$ can then be obtained by linear regression.

To estimate the error on $\Delta k_{A s: F_{A s}}$ on the calibrated value of $k_{A s: F_{A s}, F_{S i}}$ we have assumed that the statistical distribution of the measured intensities follows a Poisson law. In this case the error on the measurement of the intensity $I$ is given by $\Delta I(x)=\sqrt{I(x)}$ and the relative error is given by $\epsilon_{I}=\frac{\Delta I}{I}=\frac{1}{\sqrt{I}}$

The best fit value for $k_{A s: F_{A s}}$ can be obtained by using a weighted least squares approach (Wasserman, 2006). This is equivalent to minimize the expression :

$$
R=\sum_{i} \frac{1}{w\left(x_{i}\right)}\left[\frac{f_{A s / R B S}^{M}\left(x_{i}\right)}{1-f_{A s / R B S}^{M}\left(x_{i}\right)}-k_{A s: F_{A s}, F_{S i}} \frac{\widetilde{I}_{A s: F_{A s}}\left(x_{i}\right)}{\widetilde{I}_{S i: F_{S i}}\left(x_{i}\right)}\right]^{2}
$$

where $\left\{x_{i}\right\}$ is the set of positions where the densities have been experimentally measured. In order to favor the most precise points the weighting factors $w\left(x_{i}\right)$ have been chosen to be equal to the relative experimental error associated to $\frac{\widetilde{I}_{A s: F_{A s}}\left(x_{i}\right)}{\widetilde{I}_{S i: F_{S i}}\left(x_{i}\right)}$. 
By using error propagation laws we can then write:

$$
w\left(x_{i}\right)=\left[\frac{1}{\sqrt{\widetilde{I}_{A s: F_{A s}}\left(x_{i}\right)}}+\frac{1}{\sqrt{\widetilde{I}_{S i: F_{S i}}\left(x_{i}\right)}}\right] .
$$

The best fit value of $k_{A s: F_{A s}}$ and the associated statistical error $\Delta k_{A s: F_{A s}}$ have been calculated using the standard formula for parameter error estimation in weighted least square regression (Wasserman, 2006) and adding the contribution of the errors on RBS measurements by using error propagation formulas (Taylor, 1997).

When performing a quantitative measurement, it is important to evaluate the total measurement error. In our case three kind of errors must be taken into account:

1. The fore-mentioned statistical errors $\Delta I_{i: F_{i}}(x)$ on the measured intensities. The contribution of these errors to the arsenic mass fraction can be calculated, using standard error propagation techniques, to be

$$
\Delta_{I} \widetilde{f}_{A s}^{M}(x)=\frac{k_{A s: F_{A s}, F_{S i}}\left[I_{S i: F_{S i}}(x) \Delta I_{A s: F_{A s}}(x)+\Delta I_{S i: F_{S i}}(x) I_{A s: F_{A s}}(x)\right]}{\left[I_{S i: F_{S i}}(x)+k_{A s: F_{A s}, F_{S i}} I_{A s: F_{A s}}(x)\right]^{2}}
$$

2. The error $\Delta k_{A s: F_{A s}, F_{S i}}$ on the calibrated value of the k-factor. The contribution of this error on the arsenic mass fraction can be calculated to be :

$$
\Delta_{k} \widetilde{f}_{A s}^{M}(x)=\frac{I_{A s: F_{A s}}(x) I_{S i: F_{S i}}(x) \Delta k_{A s: F_{A s}, F_{S i}}}{\left[I_{S i: F_{S i}}(x)+k_{A s: F_{A s}, F_{S i}} I_{A s: F_{A s}}(x)\right]^{2}}
$$

3. The experimental error on the specimen thickness $\Delta t$. In this case 
it is not possible to apply the error propagation formula to evaluate the error since the dependence of $\widetilde{f}_{A s}^{M}(x)$ on $t$ is given by the previously discussed iterative calculation. In this case the contribution to the total error has been estimated using the following formula

$$
\Delta_{t} \widetilde{f}_{A s}^{M}(x)=\frac{\left|\left[\widetilde{f}_{A s}^{M}(x)\right]_{t+\Delta t}-\left[\widetilde{f}_{A s}^{M}(x)\right]_{t-\Delta t}\right|}{2}
$$

calculated for a thickness $t$.

The final error is given by:

$$
\Delta \widetilde{f}_{A s}^{M}(x)=\sqrt{\left[\Delta_{I} \widetilde{f}_{A s}^{M}(x)\right]^{2}+\left[\Delta_{k} \widetilde{f}_{A s}^{M}(x)\right]^{2}+\left[\Delta_{t} \widetilde{f}_{A s}^{M}(x)\right]^{2}}
$$

As already discussed, all dopant concentrations have been expressed using the atomic concentrations $c_{A s}^{A}(x)$. For that we have used equation 2 to calculate the absorption corrected As distribution $\widetilde{c}_{A s}^{A}(x)$ from the corrected intensities $\widetilde{f}_{A s}^{A}(x)$. The error on $\widetilde{c}_{A s}^{A}(x)$ for the case of a-SiAs have been calculated using the following formula, that can be derived using equations 10 and 2:

$$
\begin{aligned}
& \Delta \widetilde{c}_{A s}^{A}(x)= \\
& \frac{N_{A V}}{m_{A s}} \Delta \widetilde{f}_{A s}^{M}(x)\left\{\widetilde{\rho}_{a-S i A s}^{M}(x)+\widetilde{f}_{A s}^{M}(x) \frac{\widetilde{\rho}_{a-S i}^{M} \eta}{\left[1-\eta \widetilde{f}_{A s}^{M}(x)\right]^{2}}\right\}
\end{aligned}
$$

where $\eta=1-\frac{\rho_{a-S i}^{M}}{\rho_{a-A s}^{M}}$ and $\widetilde{\rho}_{a-S i A s}^{M}(x)$ is the value of the density when $f_{A s}^{M}(x)=\widetilde{f}_{A s}^{M}(x)$. 
The same procedure can be applied to obtain the concentration error to be used in the case of FinFETs. In this case using equation 7 it can be shown that:

$$
\begin{aligned}
& \Delta \widetilde{c}_{A s}^{A}(x)= \\
& \frac{N_{A V}}{m_{A s}}\left\{\widetilde{\rho}_{c-S i A s}^{M}(x)+\rho_{c-S i}^{M} \frac{m_{A s}\left(m_{A s}-m_{S i}\right)}{\left[m_{A s}-\left(m_{A s}-m_{S i}\right) \widetilde{f}_{A s}^{M}(x)\right]^{2}} \widetilde{f}_{A s}^{M}(x)\right\} \Delta f_{A s}^{M}(x)
\end{aligned}
$$

where $\widetilde{\rho}_{c-S i A s}^{M}(x)$ is the value of the density when $f_{A s}^{M}(x)=\widetilde{f}_{A s}^{M}(x)$ (see eq. 7).

\section{Results and discussion}

In this section we will present the results obtained using the previously described improved quantification technique. The discussion will be divided in two parts. First we will describe the calibration results and then we will show how we have performed dopant profiling on a FinFET test structure.

\subsection{Calibration results}

The calibration techniques described in section 2.3.3 have been applied to the a-SiAs calibration specimens described in section 2.1. Several characteristic peak families have been used: the $K$ peaks for silicon, and either the $K$ or the $L$ peaks for arsenic. The absorption correction procedure described in section 2.3.1 has been used to calculate the absorption corrected intensities 
$\widetilde{I}_{S i: K}, \widetilde{I}_{A s: K}$ and $\widetilde{I}_{A s: L}$. Then linear regression techniques have been used to calculate the value and corresponding uncertainty of:

- the k-factor $K_{A s, S i: K, K}=k_{A s: K, K}$ associated to the $K$ family of peaks of arsenic when the $K$ family of peaks of silicon is used as reference

- the k-factor $K_{A s, S i: L, K}=k_{A s: L, K}$ associated to the $L$ family of peaks of arsenic when the $K$ family of peaks of silicon is used as reference

In order to minimize the error on the k-factors we have carried out the calibration on the specimen with a $10 \%$ atomic As peak concentration. The reason of this choice can be easily understood by observing that under the fore-mentioned hypothesis that the X-ray count has a Poisson distribution the relative error is minimized for higher peak intensities. This implies a lower variance on the intensity values and a lower error on the best-fit values of the k-factors.

The comparison between the results obtained by RBS and the doping concentration measured by EDX before and after calibration of the k-factors are reported in figure 5 .

This has allowed us to obtain the following best fit coefficients for the microscope used in this experiment: $K_{A s, S i: K, K}=2.2 \pm 0.1, K_{A s, S i: L, K}=$ $1.9 \pm 0.1$.

The convergence of the iterative algorithm has also been checked by inspecting the modification of the measured concentration after several iterations (see figure 6). In our case the tolerance $\epsilon$ has been chosen to be equal to $1 \%$, to be sure that the error related to the iterative calculation was lower than the experimental error. 


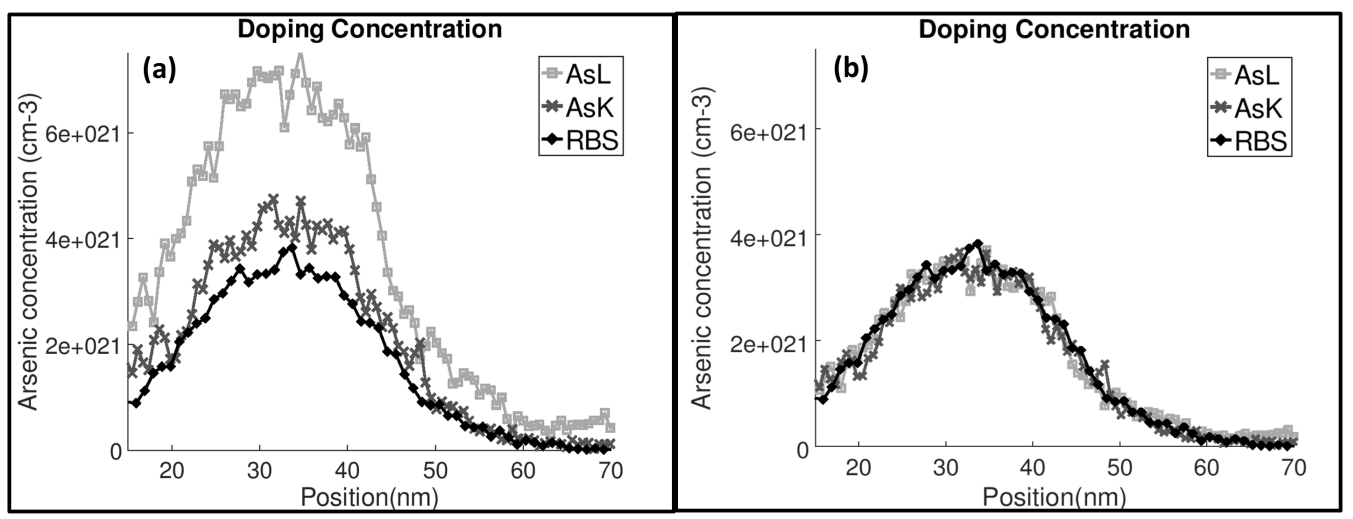

Figure 5: Plot of the measures As concentration $\widetilde{c}_{A s}^{A}(x)$ before (a) and after (b) calibration of the k-factors. In both insets the values obtained by using the $A s K$ and $A s L$ peaks are reported and compared with the values obtained by RBS. The standard values of the k-factors provided by the EDX analysis is used in (a) while the calibrated k-factors are used in (b). The absorption correction procedure described in this paper has been applied to all the profiles obtained by EDX.

It is clear from the figure that the region that is most affected by absorption effects is the peak region, and that the algorithm converges after one iteration. This can be explained by the fact that in thin specimens, such as those observed by TEM, the absorption effect is small enough to be corrected by just one iteration.

In order to check the validity of our procedure and the applicability of these coefficients to arsenic distributions with lower concentrations, we have used the previously obtained values of the k-factors to measure the doping concentration in the specimen with a $2 \%$ atomic As peak concentration. The results are shown in figure 7 where the experimental errors on the EDX measurements are also reported.

First of all it must be observed that the experimental error is larger for higher concentrations. This is due to the hypothesis that X-ray intensities 


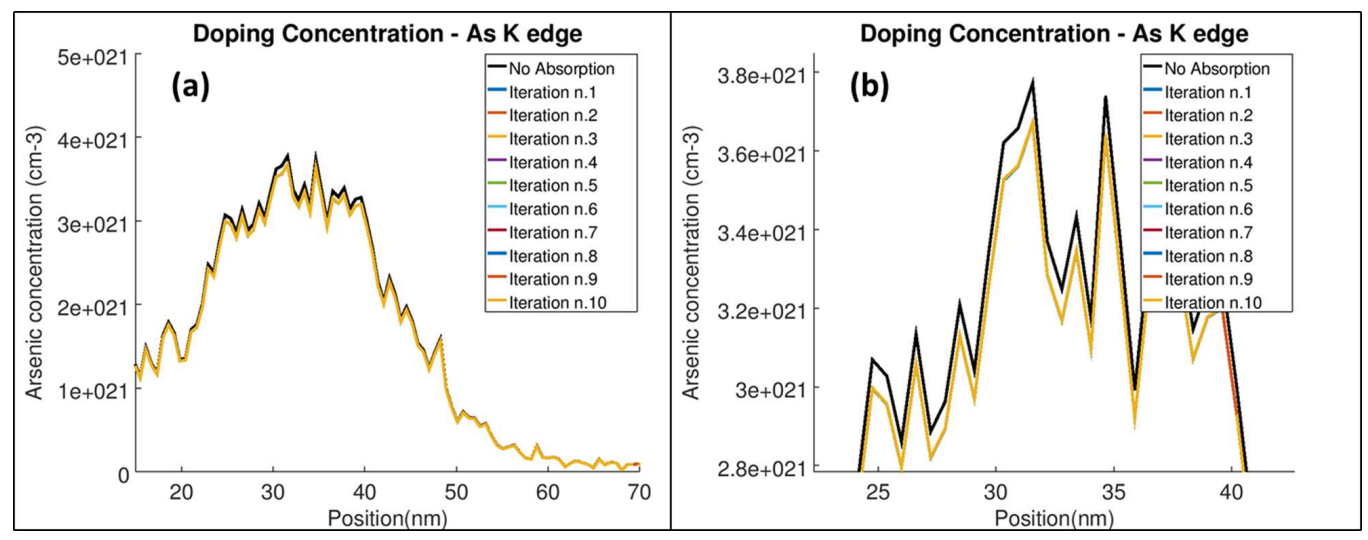

Figure 6: Absorption corrected concentrations obtained after a variable number of iterations. In (a) the full profile is shown while in (b) a zoom on the high density region is displayed. The profiles corresponding to iterations 2 to 10 are superimposed and a single curve is visible.

follow a Poisson distribution. This implies that the absolute error on the measured intensity increases with the intensity while the relative error decreases. The error on doping concentration follows the same trend since it directly depends on the error on the measured intensity. This means that, in the doping distribution peak, the measured value of doping concentration is more precise (i.e. has a lower relative error) than the values in the distribution tail, even if larger error bars appear in the graph.

Figure 7 clearly shows that the results obtained using the $K$ peaks of arsenic are more accurate than those obtained using the $L$ peaks. This is still more evident for low concentrations (i.e. in the rightmost tail of the doping profile). In the peak region there is a larger discrepancy between the AsK and RBS data that can be explained by noting that, as already discussed, the measured intensities in the peak region are affected by a larger absolute statistical variation.

The origin of this discrepancy arises from the lack of precision on the 


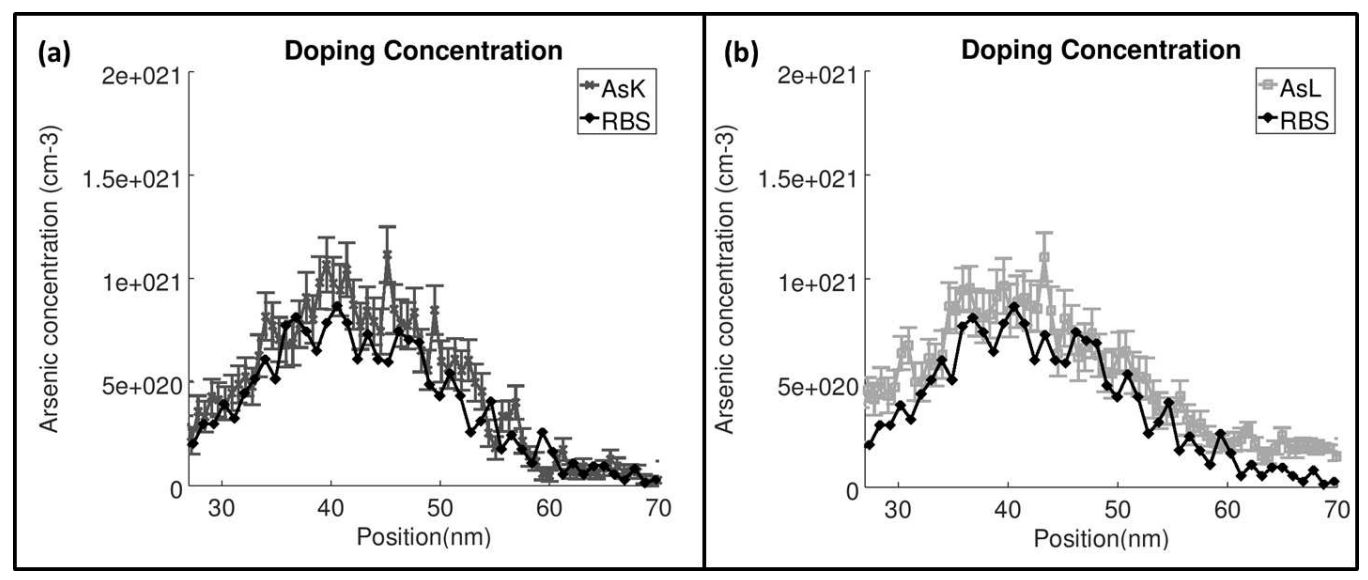

Figure 7: Plot of the measured As concentration $\widetilde{c}_{A s}^{A}(x)$ using (a) $K$ peaks for Si and As and (b) $K$ peak for Si and L peaks for As. In both cases the obtained values are compared with the values obtained by RBS.

measured intensity of the $L$ peaks of arsenic. This is due to the position of the $L$ peaks, located in the low energy region of the spectrum. In this region, the subtraction of the Bremsstrahlung noise is more difficult since the noise is higher and there are neighboring peaks associated to oxygen and carbon (Williams \& Carter, 2009). Another advantage of $K$ peaks with respect to $L$ peaks is that they are less affected by absorption effects (Williams \& Carter, 2009) even if caution must be paid to use these peaks when the observed specimens are thinned bulk specimens (MacLaren et al., 2007) and not FIB lamella as in our specific case .

For low As concentrations, such as those we are measuring this kind of problem becomes critical since the Bremsstrahlung and peak intensities can be of the same order of magnitude. This means that a small error in the the background noise interpolation has a strong impact on the measurement of As concentration. We have thus decided to use the arsenic $K$ peaks for all the measurements reported in the following. This choice can also be supported 
by the fact that K-peaks are less affected by absorption

\subsection{Doping profile in a nano-device}

To check the applicability of our method to real nanodevices we have carried out some measurements on the FinFET test structure described in section 2.1. For that an EDX hyper-map has been acquired. Some intensity maps and profiles extracted from the hyper-map are shown in figure 8 .

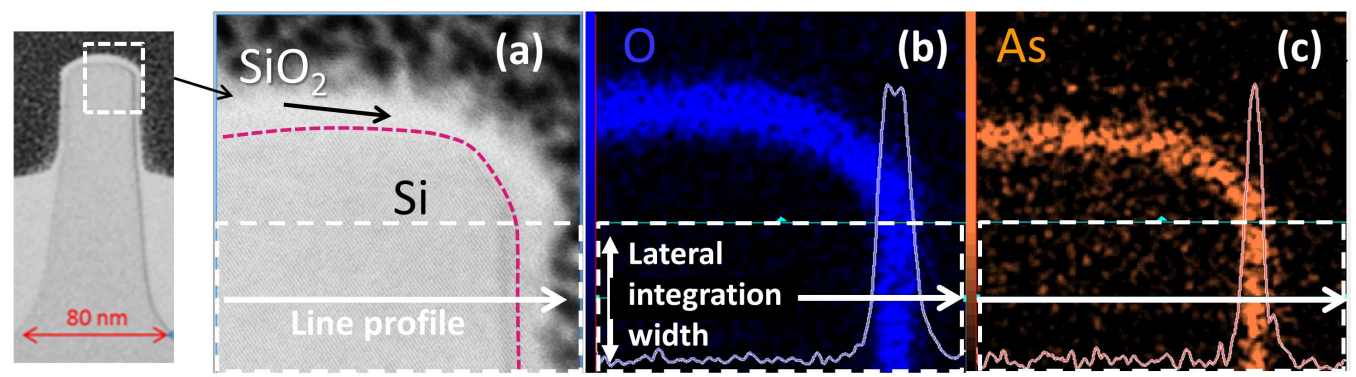

Figure 8: Bright field STEM images(a) and EDX intensity maps (b-c) obtained on a FinFET device. The $K$ peaks have been used both for oxygen and arsenic to obtain the peak intensity maps shown in (b) and (c) from the EDX hyper-map. Intensity profiles obtained using a laterally averaged line-scan are superimposed to maps ((b) and (c)). The direction and position for the line-scan as well as the lateral integration width are shown in figure (a), (b) and (c).

In order to be able to apply the same measurement geometry illustrated in section 2.3 and in figure 3 we have decided to study the distribution of one of the vertical walls of the FinFET structure and the specimen has been specifically oriented for the acquisition as in figure $9(\mathrm{a})$. The quantification method described in the previous section has then been applied to the silicon and arsenic $K$ peaks profiles. The final result is shown in figure 9 (b) where the doping profile on the lateral wall and the corresponding errors are shown. 
For density calculation, the formula of equation 7 has been used. It has to be noted, that, strictly speaking, this formula is not rigorously applicable in the peak regions, where density exceeds arsenic solid solubility in crystalline silicon (Pichler, 2004). In this regions a large fraction of the As atoms are not located in substitutional positions but they form, instead, different kind of electrically inactive clusters. At the high annealing temperature used in this work As atoms form two preferential types of clusters (see again (Pichler, 2004)): $A s_{4}$ clusters consisting of 4 As atoms placed in the four inner position of a diamond structure, and $A s_{2} V$ clusters consisting in 2 substitutional As atoms coupled with a Si vacancy. From the point of view of density calculations there is a negligible difference between subsitutional As atoms and the preferential clusters and equation 7 can still be used to describe the density in the peak region.

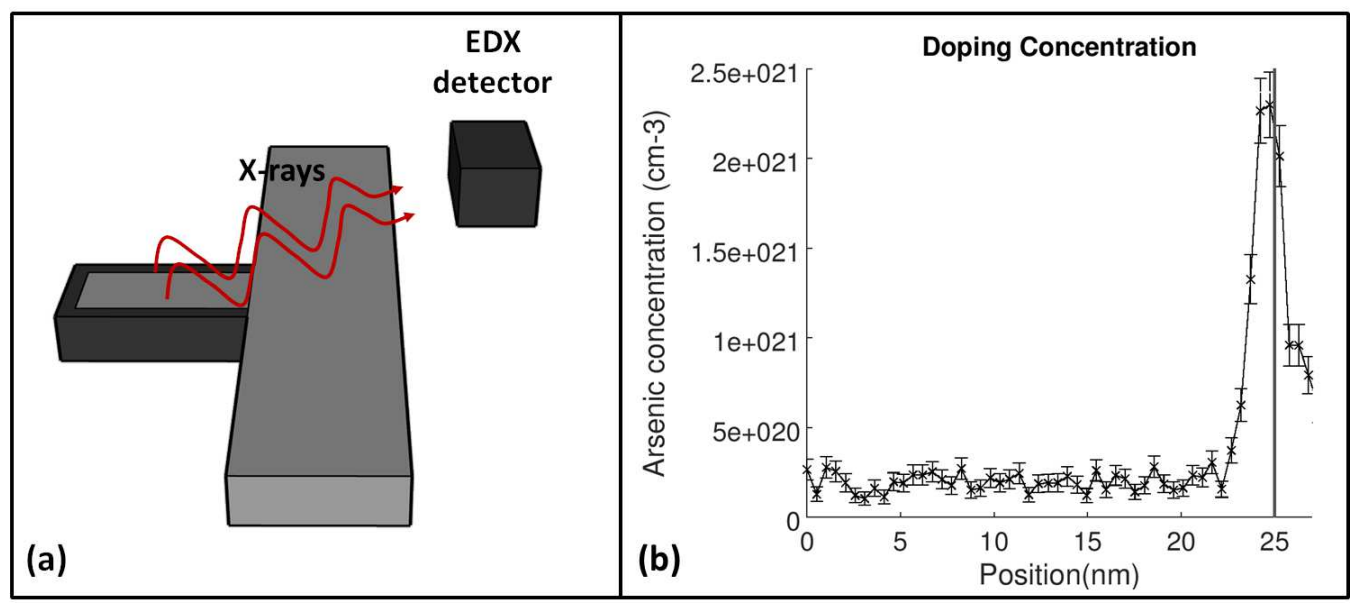

Figure 9: (a) Schematic image illustrating the geometry used to obtain the doping profile and (b) doping profile with experimental error obtained by applying the quantification method.

The results clearly show that a doping profile on a nanodevice can be 
obtained with this technique and that the obtained experimental error is low enough to be able to appreciate the concentration variations in the profile.

Finally it is interesting, in the context of doping profile measurement by STEM/EDX to try to understand what is the detection limit for arsenic concentration in a nano-device. To estimate the lowest measurable arsenic concentration we have used a simple approach based on the experimental results. It is easy to understand that a density measurement cannot be distinguished by zero if zero is included in the confidence interval (i.e. it is between $c-\Delta c$ and $c+\Delta c$ ). The obtained result obviously depends on the chosen confidence level (Taylor, 1997)(a Student coefficient equal to 1 has been chosen in this paper). The analysis of the profiles shown in figure 9 clearly shows that doping concentration is always above the concentration limit. The concentration limit can be obtained from the calibration data shown in $7(\mathrm{a})$ and can be estimated to be $7.710^{19} \mathrm{~cm}^{-3}$.

This is not an absolute estimation: the value of the detection limit clearly depends on the measured doping peaks intensity since the error on the intensity (see equation 14 and 15) can be improved by increasing the hyper-map exposition times. The formulas given in this paper can be used to calculate the error associated to a specific set of measured intensities but it is impossible to deduce a theoretical lowest limit since the maximum exposition time depends on many experimental factors such as the stability of the specimen to drift and its resistance to electron irradiation. Moreover this estimation neglects the influence of counting artifacts (such as those arising from secondary fluorescence in the specimen holder or microscope apertures) that we 
have checked to be absent in our case but could not be negligible in general.

Yet a target intensity value can be estimated. To give an example of this procedure, we have estimated the exposition times needed to obtain a given detection limit using the specific parameters of our experimental setup. More specifically we have used the values of the count per seconds measured in our setup to calculate the time needed to obtain different values of the detection limit. For the calculations we have supposed that the value of the counts-per-seconds does not change with time. The intensity rate measured in our setup amounts to $600 \mathrm{cps}$, corresponding to the total average count rate of X-rays belonging to the $K$ peaks of Si and As. The obtained detection limit variation with measuring time are shown in table 1.

\begin{tabular}{|c|c|}
\hline As density detection limit & Measuring time (live time) \\
\hline \hline $110^{19} \mathrm{~cm}^{-3}$ & 51 minutes \\
\hline $510^{18} \mathrm{~cm}^{-3}$ & $1 \mathrm{~h}$ and 43 minutes \\
\hline $110^{18} \mathrm{~cm}^{-3}$ & 8 hours and 55 minutes \\
\hline
\end{tabular}

Table 1: Estimated measuring time (live time) needed to obtain higher detection limits for a 600 cps count rate.

It can clearly be observed that in our case it is difficult to considerably improve the detection limit just by increasing the measuring times, since the presence of specimen drift, beam damage and current instability (in our case a cold Field Emission Gun gun is used) limit the measuring time to a few tens of minutes.

There is another possible way to improve the detection limit. To improve background removal, bremsstrahlung subtraction can be applied on cumulative spectra instead of pixel by pixel on the STEM/EDX map (as described in section 2.2). This can be done by creating a cumulative spectrum for each 
point of the $1 \mathrm{D}$ profile by integration in the $\mathrm{y}$ direction or by directly acquiring cumulative spectra on a 1D profile. Unluckily we were unable to test this approach due to limitations of the EDX acquisition,/analysis software we have used.

There is another possible way to improve the detection limit. Due to the limitations of the software we have used for peak integration we have been forced to apply background substract on the spectrum collected at each individual position of the STEM/EDX map (as described in section 2.2). The precision of the background subtraction can, in principle, be improved by working on a cumulative spectrum, created for each point of the 1D profile by integrating in the y direction. This possibility should be exploited when using a system capable to perform lateral integration of the spectra in a EDX map or of the direct acquisition of cumulative spectra on a 1D profile.

\section{Conclusions}

In this paper we present an improved methodology to obtain dopant profile in nanodevices. With the proposed quantification method, absorption effects can be accounted for in a rigorous way and without using in-situ beam current measurement. For that an algorithm for the self-consistent calculation of absorption effects has been proposed and the relations between the local density and the local composition of the specimen has been established using a physical model. To calibrate the model parameters, EDX and RBS results have been compared in two different specimens. The k-factors for $\mathrm{As}$ in $\mathrm{Si}$ and the associated error have been measured using linear regression tech- 
niques. The results show that a reliable calibration can be obtained by using the intensities of the silicon $K$ and arsenic $K$ peak families. The error on the measured doping concentration has also been estimated combining the different sources of experimental error using error propagation techniques. Using the proposed approach a doping profile has been obtained on a FinFET test structure with a spatial resolution on the nanometer range and a resolution limit lower than 1\%. Finally the detection limit for dopant concentration has been evaluated and the exposition times needed to improve it have been calculated using the measured count rates. It is evident from this estimation that, due to instabilities of the experimental setup, the resolution limit cannot be drastically improved by just increasing the measuring time.

\section{References}

Alvisi, M., Blome, M., Griepentrog, M., Hodoroaba, V.D., KarDuck, P., Mostert, M., Nacucchi, M., Procop, M., Rohde, M., Scholze, F., Statham, P., Terborg, R. \& Thiot, J.F. (2006). The determination of the efficiency of energy dispersive X-ray spectrometers by a new reference material, Microscopy and Microanalysis 12, 406-415.

Chu, W.K. (1978). Backscattering Spectrometry, Wei-Kan Chu, James W. Mayer, and Marc-A. Nicolet, Academic Press, 1978.

Cliff, G. \& Lorimer, G.W. (1975). The quantitative analysis of thin specimens, Journal of Microscopy 103, 203-207. 
Colinge, J.P. (ed.) (2008). FinFETs and Other Multi-Gate Transistors, Integrated Circuits and Systems, Springer US.

Custer, J.S., Thompson, M.O., Jacobson, D.C., Poate, J.M., Roorda, S., Sinke, W.C. \& Spaepen, F. (1994). Density of amorphous Si, Applied Physics Letters 64, 437-439.

EIBL, O. (1993). New method for absorption correction in high-accuracy, quantitative EDX microanalysis in the TEM including low-energy x-ray lines, Ultramicroscopy 50, 179-188.

Goldstein, J.I., Newbury, D.E., Michael, J.R., Ritchie, N.W.M., ScotT, J.H.J. \& Joy, D.C. (2018). Scanning Electron Microscopy and X-Ray Microanalysis, New York: Springer-Verlag, 4 ed.

Greaves, G.N., Elliott, S.R. \& Davis, E.A. (1979). Amorphous arsenic, Advances in Physics 28, 49-141.

HeINRICH, K.F.J. (1986). Mass absorption coefficients for electron probe microanalysis, Proc 11th Int Congr on X-Ray Optics and Microanalysis $67-119$.

Kasper, E., Schuh, A., Bauer, G., Holländer, B. \& KibBel, H. (1995). Test of Vegard's law in thin epitaxial SiGe layers, Journal of Crystal Growth 157, 68-72, URL http://www.sciencedirect.com/science/article/pii/0022024895003738.

Ke, X., Bals, S., Romo Negreira, A., Hantschel, T., Bender, H. \& 
VAn Tendeloo, G. (2009). TEM sample preparation by FIB for carbon nanotube interconnects, Ultramicroscopy 109, 1353-1359.

Kothleitner, G., Neish, M.J., Lugg, N.R., Findlay, S.D., GrogGer, W., Hofer, F. \& Allen, L.J. (2014). Quantitative Elemental Mapping at Atomic Resolution Using X-Ray Spectroscopy, Physical Review Letters 112, 085501.

MacArthur, K.E., Slater, T.J.A., Haigh, S.J., Ozkaya, D., NelLIst, P.D. \& Lozano-Perez, S. (2016). Quantitative Energy-Dispersive X-Ray Analysis of Catalyst Nanoparticles Using a Partial Cross Section Approach, Microscopy and Microanalysis 22, 71-81.

Maclaren, I., Schierholz, R., Trusty, P.A. \& Ponton, C.B. (2007). Silica Glass Segregation in 3 wt\% LiF-Doped Hot-Pressed Y2si2o7, Journal of the American Ceramic Society 90, 3307-3310.

Malis, T., Cheng, S.C. \& Egerton, R.F. (1988). EELS log-ratio technique for specimen-thickness measurement in the TEM, Journal of Electron Microscopy Technique 8, 193-200.

Morris, P.L., Ball, M. \& Statham, P. (1979). Proceedings EMAG'79 Brighton, UK Inst. Phys. Conf. Ser., vol. 52, 413.

Pichler, P. (2004). Intrinsic Point Defects, Impurities, and Their Diffusion in Silicon, Computational Microelectronics, Wien: Springer-Verlag, URL https : //www . springer . com/gp/book/9783211206874.

Qiu, Y., Nguren, V.H., Dobbie, A., Myronov, M. \& Walther, T. 
(2013). Calibration of thickness-dependentk-factors for germanium X-ray lines to improve energy-dispersive X-ray spectroscopy of SiGe layers in analytical transmission electron microscopy, Journal of Physics: Conference Series 471, 012031.

Stachurski, Z. (2015). Fundamentals of Amorphous Solids: Structure and Properties.

TAYLOR, J.R. (1997). Introduction To Error Analysis: The Study of Uncertainties in Physical Measurements, University Science Books.

Vandervorst, W., Schulze, A., Kambham, A.K., Mody, J., Gilbert, M. \& Eyben, P. (2014). Dopant/carrier profiling for 3dstructures, physica status solidi c 11, 121-129.

Wasserman, L. (2006). All of nonparametric statistics, Springer texts in statistics, New York, NY: Springer, 1st ed., 3rd print ed., oCLC: 69992043.

Watanabe, M., Horita, Z. \& Nemoto, M. (1996). Absorption Correction and Thickness Determination Using the $\zeta$-factor in Quantitative X-Ray Microanalysis, Ultramicroscopy 65, 187-198.

Watanabe, M. \& Williams, D.B. (2006). The quantitative analysis of thin specimens: a review of progress from the Cliff-Lorimer to the new $\zeta$-factor methods, Journal of Microscopy 221, 89-109.

Williams, D.B. \& Carter, C.B. (2009). Transmission Electron Microscopy: A Textbook for Materials Science, Springer Science \& Business Media. 
Ziegler, J.F. \& Biersack, J.P. (1985). The Stopping and Range of Ions in Matter, D.A. Bromley (ed.), Treatise on Heavy-Ion Science: Volume 6: Astrophysics, Chemistry, and Condensed Matter, 93-129, Boston, MA: Springer US. 